\title{
Endgültige Selbstbestimmung in Puerto Rico? Innenpolitik und US-Interessen im Vorfeld der Statusentscheidung von 1993
}

\author{
Von Ulrich Fanger ${ }^{1}$
}

Puerto Rico ist vielen Betrachtem und manchen Ländern im geographischen Umfeld als politisches Zwitterwesen lange schon ein Dom im Auge, nicht zuletzt dem benachbarten Kuba. Für die Kritiker handelt es sich um eine kunstvoll drapierte und verhätschelte Kolonie der Vereinigten Staaten; aus anderer Sichtweise gilt die Insel - inmitten einer Welt, die mit den Zwiespältigkeiten des Selbstbestimmungsprinzips ringt - als Beispielsfall für freie und autonome Entscheidungsfindung über Kulturstatut, Nationalität und Staatlichkeit.

Im Abstand von 26 Jahren wurde die Wählerschaft Puerto Ricos zweimal zum Volksentscheid über den Status der Insel aufgerufen, nachdem seit 1898 bereits sechs Abstimmungen zu dieser Frage und zusätzlich Referenden in den Jahren 1970 und 1991 durchgeführt worden waren. Das jüngste Votum am 14. November 1993 hat den Bestrebungen zur Eingliederung in die USA (als 51. Bundesstaat) zum zweitenmal in einer Generation eine Absage erteilt, so daß der staatsrechtliche Grundsatzdisput nun vom Tisch ist, jedenfalls auf absehbare Zeit. Im Ergebnis stimmten 48,5\% für die Beibehaltung der gegenwärtigen Sonderstellung, 46,3 \% furr die Eingliedenmg, und nur 4,4\% sprachen sich für staatliche Eigenständigkeit aus. Die Wählerbeteiligung in Puerto Rico liegt mit zwischen $70 \%$ und $85 \%$ traditionell weit über der jedes Wahlkreises in den kontinentalen USA, und die bei der Volksabstimmung erzielte Partizipation von 73,6\% läßt jedenfalls keinen Zweifel an der "volonté générale" der Inselbevölkenung zu. Allerdings ist zu vermerken, daß weder die eine noch die andere der beiden Hauptaltemativen mehr als die Hälfte der Stimmen erzielen konnte und der Vorsprung der Mehrheit äußerst knapp bei 37.399 Stimmen lag. Vergleicht man die Abstimmungslage mit der von 1967, so ist festzustellen, daß die Integrationisten in der langfristigen Tendenz Boden gewonnen haben; Argumente für eine spätere Wiederbelebung der innenpolitischen Debatte sind also gegeben.

Wie ist der emeut bestätigte Status des Territoriums vor dem Hintergrund seiner politischen Entwicklung zu bewerten, welche Kräfte und Interessen standen sich in der Statusfrage gegenüber, und welche politischen Einflüsse gingen und gehen von den USA aus, um eine definitive Festschreibung der puertoricanischen Staatlichkeit zu bewirken?

1 Eine stark gekürzte Fassung dieses Beitrages findet sich bei G. Rieger (Hrsg.), Die Karibik zwischen Souveränität und Abhängigkeit, Freiburg 1994, S. 85-95. 


\section{Die politische Entwicklung zum assozilerten Freistaat}

Bis zur Besetzung der Insel durch amerikanische Truppen im spanisch-amerikanischen Kriey am 25. Juli 1898 wies die Geschichte Puetto Ricos zahlreiche Parallelen zur Entwicklung Kubas auf. Puerto Rico wurde im 19. Jahrhundert von Havanna aus als kolonialspanischer Verwaltungsbezirk regiert, und beide Inseln waren - nach fast vierhundert jähriger Kolonialzeit - die letzten spanischen Territorien in Amerika. Anders als in Kuba war aber hier die nordamerikanische Inbesitznahme direkt und umfassend, und das Streben nach nationaler Identität und Eigenständigkeit gedieh nicht über vorsichige Ansätze hinaus, mit denen bis in die jüngste Zeit jeweils nur ein unbedeutender Teil der öffentlichen Meinung für die staatliche Unabhăngigkeit mobilisiert werden konnte. Allerdings war der gesamte Verlauf der Beziehungen zu den Vereinigten Staaten von einem Ringen um erweiterte politische Rechte und Zustăndigkeiten unterhalb der Schwelle zur Eigenstaatlichkeit geprägt, und unter der amerikanischen Oberhoheit entwickelten sich schlieBlich verschiedene denkbare staatsrechtliche Lösungsvorstellungen, die die innerpolitische Auseinandersetzung nach dem Ende des ersten Weltkrieges bestimmt haben. Seit der Obertagung der USStaatsbürgerschaft an die Inselbevölkenung (1917) wurde ihr schlieBlich etuypenweise eine eingeschrănkte politische Selbstverantwortung übertragen, in einem ProzeB, der erst unter der Regierung Truman ab 1950 (Act of Congress/Public Law No. 600) einen klaren rechtlichen Status festgelegt hat. Puerto Rico ist seither ein mit den Vereinigten Staaten verbundenes Sonderterritorium unter der Bezeichnung eines "assoziierten Freistaats" (Estado Libre Asociado/Commonwealth). Es handelt sich dabei um den intemationalen Sonderfall eines hochentwickelten assoziierten Staatswesens, das völkerrechtlich als "Staatsfragment" einzuordnen ist ${ }^{2}$.

In Volksabstimmungen fanden 1951 und 1952 sowohl die staatsrechtliche Neuordnung als auch der Erlaß einer eigenen Verfassung breite Zustimmung3. Die Eigenart des hier gefundenen Rechtszustands ist seither immer wieder zum Ansatzpunkt für interne und intemationale Diskussionen über die endgültige Definition der puertoricanischen Staatlichkeit geworden. Mit der Errichtung der UNO gerieten die USA denn auch in die für sie äußerst unangenehme Lage, dem Treuhandausschuß der Weltorganisation jährlich Rechenschaftsberichte über die politischen, wirtschaftlichen und sozialen Bedingungen auf der Insel vorlegen zu müssen. Seit dem zweiten Weltkrieg entstand somit ein enger Zusammenhang

Vgl. Kirchner, K.-F.: Die Rechtsstellung des Commonwealth of Pueno Rico, Heidelberg 1967, der die völkerrechtliche Definition nach Jellinek und Hatschek referien.

3 Im Referendum am 04.06.51 stimmten 76,1\% der Wähler für die Annahme des Statusgesetzes (Law 600), bei einer Wahlbeteiligung von 65\%. Die Verfassungsabstimmung am 03.03.52 fand $81,9 \%$ an befürwortenden Scimmen und 18,1 \% Ablehnung; bei einer Wahlbeteiligung von nur $59 \%$ erreichte die Zustimmung allerdings nicht die einf ache Mehrheit der Wahlbeteiligten. Quelle: Govemment of Puerto Rico: Notes and Comments on the Constitution of the Commonwealth of Puerto Rico. San Juan 1952, S. 28-30. 
zwischen dem Ansehen der USA vor dem Weltforum und der Bereitschaft der nordamerikanischen Seite, auf puertoricanische Belange einzugehen. Den Makel einer Protektoratsmacht komnten die USA 1953 zunächst abstreifen, als sich nach dem Verfassungsreferendum von 1952 in der Weltorganisation der BeschluB durchsetzen lieB, daB Puerto Rico nunmehr als "selbstverwaltetes Territorium" einzustufen sei und somit den UN-Resolutionen entsprach.

Im Innenverhältnis zwischen Puerto Rico und den USA war aber auch in der Zwischenzeit der Dialog uber weitere Veränderungen, Lockerungen und wirtschaftliche Sonderkonditionen fortgefuhrt worden. Er bezog seine wesentlichen Anstöße aus dem Wählerverhalten der Inselbevölkenng, und speziell aus der relativen Stärke bzw. Schwäche derjenigen Gruppen, die für eine Unabhängigkeit der Insel eintraten. Ein Ergebnis war die Zusichenmg der Regienung Eisenhower (November 1953), daB die USA in Zukunft jedwede auf freier Abstimmung fußende Statusentscheidung der gewählten Vertretungskörperschaften Puerto Ricos oder der Wählerschaft selbst anerkennen würden.

Der im Dezember 1960 von der UdSSR der UN-Vollversammlung vorgelegte EntschlieBungsantrag fir eine "Doclaration on the Granting of Independence to Colonial Countries and Peoples" (Resolution 1514/XV) fuhre ein Jahr später zur Gründung des UNEntkolonisienmgs-Ausschusses. Dies setzte eine neue Runde von Puerto-Rico-Debatten in Gang, sowohl in den USA wie vor den internationalen Gremien. Zunächst verfügte die Regienmg Johnson 1964 die Einsetzung einer gemischten Statuskommission (mit sieben amerikanischen und sechs puerroricanischen Mitgliedem) zur Úberwachung der besonderen Bindungen zwischen den beiden Partnem. Úber mehrere Jahre konnten die USA eine Untersuchung des Falls Puerto Rico seitens des neuen UN-Ausschusses durch diplomatische Aktivitäten verhindern. Auf Antrag Kubas wurde 1966 erstmals die UN-Vollversammlung mit der Frage befaBt, und mit der großen Zahl der jungen entkolonisierten Staaten beschloB die Mehrheit, Puerto Rico falle in den Zuständigkeitsrahmen der Resolution 1514. Diese Entschließung gab den AnstoB zur Durchführung der Volksabstimmung von 1967, das erste Votum, das explizit die völkerrechtliche und politische Zukunft der Insel zum Inhalt hatte und eine knappe 2/3-Mehrheit fur den Commonwealth-Status erbrachte. Der damit erreichte und aus der Sicht Washingtons dauerhafte Zustand einer harmonischen Beziehung wurde jedoch 1971 gestört, als die Statusfrage emeut vom Entkolonisierungsausschuß aufgegriffen und auf kubanische Initiative zum regelmäBigen Verhandlungsgegenstand gemacht wurde 4 . Der Ost-West-Konflikt in seiner karibischen Dimension warf

4 Die völkerrechtliche Handhabe für die emeute Befassung mit der Puerto-Rico-Frage war die Feststellung, daB das Referendum von 1967 ohne Beteiligung der Unabhängigkeitsbefürworter durchgeführt worden war (diese Gruppen hatten zum Abstimmungsboykott auf gerufen und nur 0,6\% an Proteststimmen verzeichnen können), und daB der Volksentscheid ohne intemationale Beobachtung abgelaufen war. Das Ergebnis wurde daher nicht als völkerrechtlich bindend anerkannt und die endgültige Lösung von einem weiteren ordnungsgemäßen Votum abhängig gemacht. 
seinen Schatten auf die Beziehung zwischen den USA und dem Inselterritorium. Der Fall Puerto Rico wurde ab 1972 nahezu jährlich Gegenstand eines zähen diplomatischen Ringens zwischen den USA - die selbst nicht AusschuBmitglied waren - und den 22 in ihm vertretenen Staaten. Tatsächlich handelte es sich um eine Verlagerung des kalten Krieges zwischen Kuba und den USA auf das intemationale diplomatische Parkett; die Schärfe dieser Auseinandersetzung kann als eine der Erklärungen für Washingtons nachhaltige Härte in der Haltung zu Kuba gelten. Bis 1976 gelang es den USA in der Regel, die Ablehnung der Anträge oder eine Vertagung zu erreichen. Trotz massiver Beeinflussungsversuche ging 1976 erstmals die Stimmenmehrheit verloren (10:10, bei zwei Enthaltungen), 1978 erfolgte dann ein Votum (mit $10 \mathrm{zu} 12$ Enthaltungen), das implizit eine Verurteilung der USA enthielt und von Kuba als Triumph uber den Erzfeind gefeiert wurde. Ähnliche Resolutionen des UN-Ausschusses kamen in den Folgejahren zustande. Die Bestrebungen der USA richteten sich seither darauf, die Oberfuhnmg des Themas auf die Agenda der Vollversammlung zu verhindem.

Die zwanzigjährige Zeitspanne von der staatsrechtlichen Neuordnung von 1953 bis zur Wiederaufnahme der internationalen Statusdebatte (1972) deckt sich nahezu mit der eigentlichen Wachstumsphase der puertoricanischen Wirtschaftsmodells, die ihren Hauptantrieb von der sog. Operation Bootstrap ("Stiefelschlaufe", verwendet etwa im Sim von: "Sich an den eigenen Stief eln aus dem Sumpf ziehen".) des ersten von der Inselbevölkerung direkt gewählten Gouverneurs L. Muñoz Marín bezogen hat. Puerto Rico hat durch die in diesem Rahmen verfolgte Strategie der Industrieentwicklung eine eindeutig auf den Binnenmarkt der USA orientierte Ausrichtung erhalten; seine Handelsstruktur nähert sich der einzelner amerikanischen Bundesstaaten.

Neben der ausländischen Unterstützung für die Unabhängigkeitsidee war es die wachsende Militanz der zahlenmäßig kleinen Gruppen von Befürwortem der Unabhängigkeit (abgesehen von den beiden of fiziellen Partido Independentista/PIP und Partido Socialista: die Untergrundbewegungen FALN und Boricua People's Army)5, die die Statusdebatte seit

5 Zwischen 1970 und 1983 hatten sich kleine militante Gruppen von PIP und PSP abgesonden, die den für sie aussichtslosen WahlprozeB grundsätzlich ablehnten und den bewaffneten Kampf verfolgten. Dazu gehören vor allem das Ejército Popular Boricua (Boricua People's Army, auch: "Macheteros"), die mit Anschlägen auf US-Großuntemehmen und militärische Einrichtungen vorgingen (1981: Sprengung von neun Flugzeugen der amerikanischen Nationalgarde) und die FALN (Fuenas Armadas de Liberación Nacional Puenoriquena), die auf dem Festland 1985/86 mit rund 100 Bombenattentaten und mit der Emondung Präsident Reagans drohten. Konzertiente Fahndungsaktionen des FBI und der puertoricanischen Polizei führten zur Aburteilung bzw. bei Gefechten zum Tod von Terroristen und zur Zerschlagung der Kommandos. Nach dem Stand von 1991 befanden sich 19 verurteilte Attentäter in den USA in Haft. Aus dem kubanischen Exil der Restgruppen fanden seit Ende der Achtzigerjahre keine weiteren Ubergriffe statt. Als "legaler Arm" der marxistisch-leninistischen "Liga Socialista Puenoriquena (ISP) und der FALN in den USA ist das Movimiento de Liberación Nacional Puertoriqueno (MLN) mit Sitz in Chicago tätig. 
Mitte der 70er Jahre wieder aufleben ließ. Dabei waren hier die Abstimmungen vor dem UN-EntkolonisierungsausschuB, aber auch Resolutionen mit Fordenmgen nach puertoricanischer Unabhängigkeit von seiten der Bewegung der Blockfreien und der Sozialistischen Intemationale (1983) von Einfluß. Dennoch verlief die Diskussion uber den "Kolonialstatus" der Insel und seine Altemativen bis in die 80er Jahre weitgehend abgehoben von der Meinungsbildung im Lande selbst. Der Unabhăngigkeitsbewegung wurde auf intemationaler Ebene die Rolle einer politischen Kraft zugemessen, die ihr in Puerto Rico selbst versagt geblieben ist; ihr Wählerreservoir ging nie uber rd. $6 \%$ hinaus, und in den letzten Jahren konnten jeweils nur 3-4\% der Bevölkerung fur das Anliegen mobilisiert werden. Wie der Wahlboykott dieser Gruppen 1967 gezeigt hat, sind sie sich ihrer geringen Resonanz vollauf bewußt und erklärten deshalb bislang regelmäßig jeden Wahlvorgang in Puetto Rico zur "Wahlfarce". Man könnte dies als die typische Schmollhaltung notorisch schlechter Verlieter abtun, wäre dahinter nicht das alte marxistische Vorhut-Denken verborgen. Nach dieser Vorstellung ist die Wählermasse entweder noch zu ungebildet, um ihrem "echten" Willen" legitim Ausdruck geben zu können; oder sie orientiert ihre Entscheidung unrechterweise an ihren "engstimigen materiellen Interessen" - in diesem Fall also an der Wahrung des erworbenen Versorgungsstandes. Nur die "Avantgarde" sei demnach legitimiert, dem Volkswillen Ausdruck zu geben.

Das Störfeuet von dieset Seite ist ohne großen Einfluß geblieben; unabhängig davon ist aber die Statusdiskussion emeut in den Vordergnund geruckt worden durch Zweifel an der Beständigkeit des Wirtschaftsmodells in Auswirkung der Weltwirtschaftskrise seit 1980. Sie hat dann vor allem den Wahlkampf 1988 völlig beherrscht.

\section{Die Vertretung der Statusinteressen in der Parteienlandschaft Puerto Ricos}

Auf den ersten Blick erscheint es so, als wenn sich das puertoricanische Parteiensystem in ein Pendant zur parteipolitischen Struktur der kontinentalen USA entwickelt habe. Nach mehreren Jahrzehnten einer Dominanz der Gründerpartei PPD (Partido Popular Democrático, mit sozialdemokratischer Tendenz) hat man es seit rund 20 Jahren de facto mit einem Zweiparteiensystem zu tun, das aus den beiden Blöcken des (eher liberal-konservativen) PNP (Partido Nuevo Popular) und des PPD besteht, die sich als Volksparteien konsolidiert haben, zusammen durchgängig 90-95\% der Wählerstimmen auf sich vereinigen, und zwischen denen es durch Bewegungen einer relativ kleinen Wechselwählerschaft periodisch zu Regierungswechseln kommt.

Die programmatischen Vorstellungen dieser Gruppen kommen in der "Gemeinsamen Erkläning der Macheteros und der FALN" vor dem Dekolonisieningsausschuß der UNO vom August 1988 zum Ausdruck (in deutscher Ubersetzang abgedruckt in: Archiv für transatlantische Sozial- und Kulturgeschichte (Hrg.): PuertoRicolibre - Kolonie der USA?, Bremen 1992, S. 32 f.). 
Trotz enger gewordener Verbindungen zu den großen US-Parteien, haben sich diese beiden Gruppen jedoch nicht zu puertoricanischen Klientel-Anhängseln der Republikaner bzw. der Demokraten entwickelt. Deren Interesse an Puerto Rico beschränkt sich vielmehr weitgehend auf die Durchfuhnung der Kandidatenvorwahlen (primaries)6, deren Ergebnisse im inneramerikanischen Wahlkımpf den Wert der Demonstrationswirkung zeitigen können. Tatsächlich hat sich die Programmatik der Parteien in Puerto Rico stets an den beiden Streitpunkten politischer Status und marktwirtschaftlich-kapitalistische Wirtschaftsordnung orientiert und verläuft häufig nicht im Einklang mit der Meinungsbildung in den Festlandsparteien.

Deutlich akzentuiert wurde der unterschiedliche Zielkatalog zwischen den befreundeten Parteien beispielsweise, als die Demokratische Partei der USA noch vor den puertoricanischen Wahlen von 1980 die Möglichkeit einet unabhängigen staatlichen Entwicklung einräumte, damit die Position der ihr nahestehenden PPD schwächte, die traditionell für die Beibehaltung des Commonweslth-Modells eintritt, und damit Flügelkămpfe in dieser Partei auslöste. Ähnliche Dissonanzen löste der SprachenerlaB der PPD-Regienung (April 1991; Einführung des Spanischen als alleinige Amtssprache) bei den Demokraten im Kongreß der USA aus.

Insgesamt gesehen kristallisieren sich in den beiden Großparteien die unterschiedlichen Zielvorstellungen zum Verhälonis gegenüber den USA in der Weise, daß PNP den Beitritt zur Union (Statehood/Estadidad) verfolgt und dabei die Interessen eines Teils der unternehmerischen Oberschicht (insbesondere des Handels) vertritt und zugleich auf breite Unterstützung durch die unteren Bevölkenungsschichten und die organisierte Arbeiterschaft setzen kann?. Das Votum der ärmeten Schichten ist dabei vor allem an die Erwarnmg erhöhter sozialer Transferleistungen aus den USA gebunden. PPD tritt demgegenüber für die Beibehaltung des von dieser Partei seinerzeit durchgesetzten Sonderstatus des assoziierten Freistaates ein; sie hat dadurch - abweichend von der demokratischen Partei in den USA - weitgehend auf den Rückhalt in der Arbeiterschaft und der Gewerkschaftsführung verzichten müssen, die eine US-Integration stets mit der Aussicht auf verstärkte arbeits-

6 Die puertoricanische Bevölkenung hat kein Wahlrecht bei den Kongreß-, Senats- und Präsidentschaftswahlen auf dem Festland, da dieses mit der Bundeseinkommensteuer gekoppelt ist, der PuertoRico nicht unterliegt. Die beiden Parteien der USA führen aber vor Präsidentschaftswahlen seit 1980 auch auf der Insel "primaries" durch, zu deren Teilnahme die Sympathisanten aufgerufen werden. Sie werden in der Regel von Teilgruppen oder Führungspersönlichkeiten aus den Reihen von PNP und PPD organisien, ohne daß die puentoricanischen Parteien als solche of fiziell involvien werden.

7 Bei der Neubildung des PNP nach 1967 hat sich der gegen eine US-Integration eingestellte großagrarische Sektor (insb. Zuckeranbau und-verarbeitung) abgespalten. Die Partei vertrat seither vornehmlich die "progressive Industriebourgoisie" und die städrischen Mittelschichten. Vgl.: Melendez, Edgardo: "The social basis for Annexionism in Pueno Rico and the Emergence of the New Progressive Party", in: Revista Homines (San Juan) Bd. 11 (1987-88) 1/2, S. 49-81 
rechtliche Freiheiten und Leistungen verbunden hat ${ }^{8}$. Allerdings hat PPD während ihrer beiden letzten Amtszeiten 1984-92 unter Gouvemeur Hemandez Colón auf Druck des linken Flügels versucht, den Rahmen des Assoziienungsvertrages mit kleinen Forderungsschritten und gewagten Initiativen in Richtung einet quasi-Souverănităt zu erweitern ${ }^{9}$. Als Ziel schälte sich hier die Idee einer Staatlichkeit heraus, die - sarkastisch als "Jibaro - Statehood 10 " glossiert - schließlich alle Vorteile des amerikanischen Wirtschafts- und Sozialsystems mit einem beständig auszuweitenden Rahmen eigenständiget intemationalet Präsenz Puerto Ricos verbunden hätte. Das Motto für die Abstimmungskampagne 1993: "Para lo mejor de dos mundos" wurde somit damals schon vorweggenommen. Die PPD-Regierung hat damit in den USA größere Widerstände und Zweifel wachgerufen als sie jemals zuvor bestanden. Die Mitgliedschaft des PPD hatte ursprtinglich ein breites Spektrum von Interessengruppen umfaßt, von konservativen Befurwortem einer engstmöglichen Bindung an die USA bis hin zu "Nahezu-Independentistas", die bis auf die gemeinsame Währung und die Außenverteidigung alle Bindungen kappen wollten. Ihre breite Unterstutzungsbasis einer Sammlungsbewegung der funfziger Jahre hat die Partei jedoch inzwischen verloren und repräsentiert mit ihren Zielvorstellungen nunmehr in etster Linie einen großen Teil der Mittelschichten, die in der Landwirtschaft Tatigen (wegen Befurchtungen vor einer noch gnadenloseren Konkurrenz im Fall der Eingliedenung) und einen Teil des akademischen Sektors (insbesondere Lehrerschaft und Angestellte), der stark kulturpolitisch engagiert ist und sich gegen eine bedrohliche anglophone Oberfremdung zu wehren sucht.

Im Vergleich zur Programmatik der beiden großen Parteien hat die Befurwortung der Unabhängigkeit uber die letzten Jahrzehnte hin einen gleichbleibend niedrigen Zustimmungsgrad erreicht. Die Organisationen dieses Spektrums verbinden großenteils mit der Unabhängigkeit marxistische Zielsetzungen, schließen aber (mit dem PIP) auch Gruppen sozialdemokratischer Nationalisten ein, die sich weitgehend aus der Kulturszene und der Akademikergemeinde rekrutieren und deren Forderungen sich vordergrutudig auch auf die Wahrung kultureller Autonomie und auf ökologische Programmpunkte richten. Den

8 Vgl. Nohlen, D.: Puerto Rico, in: Nohlen, D./F. Nuscheler (Hrsg.), Handbuch der Dritten Welt, Bd. 3, Bonn 1992, S. 638

9 Bereits während seiner ersten Amtszeit als Gouvemeur war Rafael Hemandez C. 1973-76 mit seinem Versuch gescheitert, dem Kongre $B$ in Washington für eine weitreichende Autonomieausweitung im Rahmen der bestehenden formalen Assoziierung abzuringen. Emeute Versuche, durch außenpolitische Alleingänge den Zuständigkeitsrahmen zu erweitem, wurden 1986 vom Kongreß blockien (s. Latin American Weekly Report vom 02.05. und 28.08.1986). Das Scheitem dieser Strategie und seine Niederlage in dem von ihm anberaumten Ref erendum (Dezember 1991, über ein Verhandlungspaket zur Festschreibung demokratischer Grundrechte) bildeten dann den Hintergrund für seinen Rückzug aus dem politischen Leben Ende 1992.

10 Jibaro = ursprünglich puertoricanischer Gebirgsbauer, zugleich häufig als landsmannschaf tliche Bezeichnung verwendet. Vgl. Fanger, $U$.: "Puerto Ricos Assoziienung mit den USA - Chancen und Grenzen autonomer Entwicklung in Abhängigkeit", in: Nuhn, H. (Hrsg.): Krisengebiet Mittelamerika, $\mathrm{Br}$ aunschweig 1985, S. 255-266. 
Independentistas ist es einerseits nicht gelungen, größere Breitenwirkung zu erzielen, andererseits wurde ihr Rückhalt mit dem Zusammenbruch des sozialistischen Lagers und durch die Entwicklung in Kuba auch nicht in nennenswertem Umfang geschmälert.

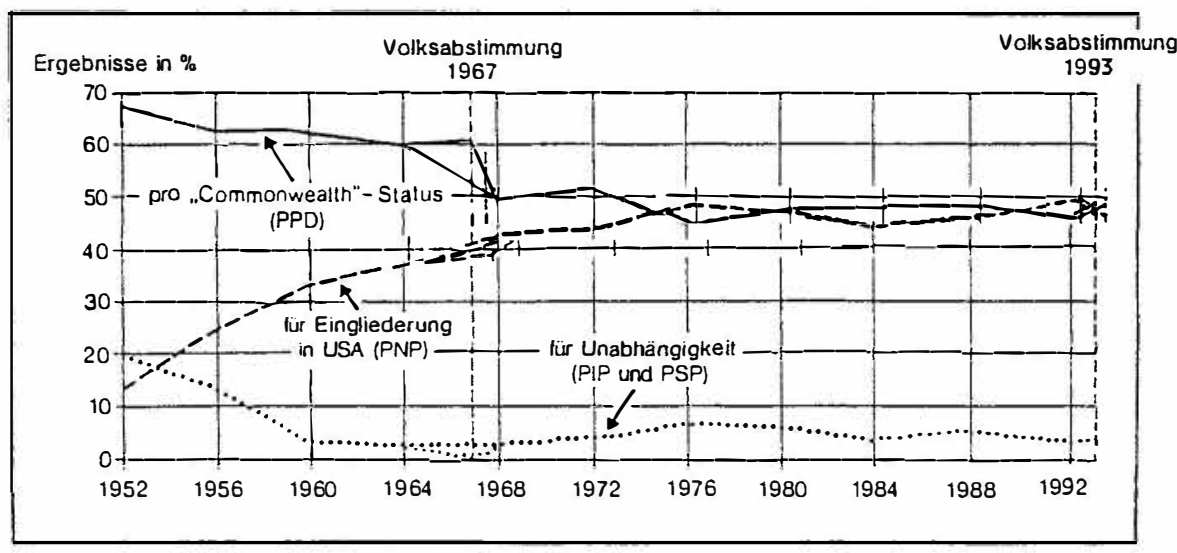

Wählerverhalten in der Frage des politischen Status Puerto Ricos Quelle: Daten der Junta Estatal de Elecciones

Puerto Rico als Bundesstaat? - Verãnderte Inter essenlage und schwankendes Engagement auf seiten der USA

Seit der Politik imperialer Expansion unter Theodore Roosevelt war für die USA Puerto Rico zuallererst aus strategisch-militärischen Uberlegungen von Bedeutung, mit Hinblick auf eine unmittelbare Präsenz im karibischen Raum und die Sicherung des Seegebiets 11. Trotz Weiterbestehens des Konflikts mit Kuba verliert dieses Faustpfand seit dem Ende des Ost-West-Konflikts seine strategische Dimension. Nach dem zweiten Weltkrieg war die Vorstellung hinzugetreten, daß man aus der Insel einen Beispielsfall winschaftlicher und gesellschaftspolitischer Entfaltung für den gesamten Entwicklungskontinent Lateinamerika

11 Eine eingehende Darlegung der nordamerikanischen Regionalstrategie findet sich bei Sandner, Gerhard: Zentralamerika und der Feme Karibische Westen. Stuttgan 1985, S. 169 ff 
machen könne. Tatsächlich hat Puerto Rico in den 50er Jahren eine Zeitlang eine Ausstrahlungswirkung und Vermittlerfunktion im hispano-amerikanischen Raum wahmehmen können; besonders hat sich dies in der Oberuagung organisatorischer Impulse auf den Gebieten staatlicher Verwaltungsorganisation, des Rechtswesens und im Aufbau von Parteien und Gewerkschaf tsverbänden niedergeschlagen. Auch hier ist aber etwa seit 1965 ein Bedeutungsverlust eingetreten - eine Folge der beginnenden Eigendynamik süd- und mittelamerikarischer Länder, in Verbindung mit der bilateralen Auslandshilfe der ersten Entwicklungsdekade.

Abgesehen vom strategischen Wert beruhte die Bedeutung Puerto Ricos für die Vereinigten Staaten seit jenem Zeitpunkt daher zunehmend auf drei Interessenbereichen: (1) dem Nutzen als assoziierter Billiglohn-Standort fir Industrien im Zoll- und Steuerpräferenzsystem, (2) dem außenpolitischen Wert der Modellwirkung als Experimentierfeld marktwirtschaftlich-industrieller Entwicklung im Vergleichsrahmen der anderen überbevölkerten Inselstaaten, und (3) der Rückwirkung eines stabilisierten politischen Systems auf die puertoricanische Diaspora auf dem Festland und daruber hinaus auf die wachsende hispanische Wählerschaft in den USA insgesamt.

Inzwischen sind die beiden erstgenannten Aspekte seit 1980 ihrerseits einem Bedeutungsschwund unterworfen, und zwar einmal durch die Verwirklichung der Caribbean Basin Initiative (CBI; Eröffnung konkurrierender Billiglohn-Standorte); im zweiten Fall ist die Modellwirkung stark durch die anhaltend krisenhafte Entwicklung der puertoricanischen Wirtschaft insgesamt beeintrăchtigt worden.

Demgegenüber hat die innenpolitische Wechselwirkung zwischen den Politiksystemen Puerto Ricos und der USA ihren Stellenwert behalten oder sogar an Bedeutung gewonnen. Sie ist es, die in ihrer Verzahnung mit der internationalen Statusdebatte zunehmend die Haltung der US-Regierung und der Festlandsparteien bestimmt hat, und sie hat die jüngsten Entwicklungen in der Statusfrage hervorgebracht.

Mit dem auf Präsident Nixon zurückgehenden Vorschlag zur Ausarbeitung eines neuen Vertragswerks unter dem Titel "Compact of Permanent Union" reagierten die USA relativ früh auf die skizzierten Entwicklungen in Puerto Rico und im intemationalen Umfeld. Unter Zugrundelegung des von der Regienung in Auftrag gegebenen sog. Tobin Report suchte die Nachfolgeregierung Ford diese Initiative 1976 in eine dauerhafte Regelung zu übertragen. Sie war inhaltlich weitgehend auf die Forderungen und innenpolitischen Bedürfnisse der damaligen puertoricanischen Regierungspartei PPD zugeschnitten, geriet aber in das Kreuzfeuer der beiden Oppositionsparteien auf der Insel. Nach dilatorischer Behandlung im Kongreß wurde der Versuch eines Neubeginns schließlich das Opfer einer zweif achen Verschiebung des politischen Terrains: in Puerto Rico siegte 1976 erstmals die 
PNP - Verfechterin det Integration -, und der Wahlsieg der Demokraten unter Carter leitete Anfang 1977 eine distanziertere Politik gegenüber Puerto Rico ein.

Sowohl unter Carter wie unter Reagan ist die Haltung der USA zu Puerto Rico von einer gewissen Scheu vor definitiven Festlegungen gekennzeichnet gewesen. Wechselnde Aussagen beider Präsidenten zur Erwonschtheit der staatlichen Eingliederung Puerto Ricos bzw. zur Beteitschaft der USA, gegebenenfalls auch ein Unabhängigkeitsvotum anzuerkennen, kennzeichneten die zunehmend abwartende Haltung, die vor allem in beiden Parteilagem im Senat und auch im Repräsentantenhaus eingenommen wurde. Präsident Reagan, der sich im Januar 1982 noch klar zum "integralen Anschluß der Insel" bekannt hatte, änderte - offenbar unter dem Eindruck der Terroranschläge der Machetero-Gruppe 1985/86 - seinen Entschluß und entzog der puertoricanischen Rechten seine bedingungslose Unterstiitzung 12 .

Die Meinungslage in den beiden Parteilagem des Repräsentantenhauses und des Senats war seit dem Referendum von 1967 durch ein Klima vorsichtiger Distanzierung von der Eingliederungsidee gekennzeichnet; allerdings weisen die beiden Parteien unterschiedliche Grundstimmungen in ihrer Mitgliedschaft auf (vgl. dazu unten). Gründe für die Haltung der Abgeordneten muß man in den mittlerweile sehr schwerwiegenden Integrationsproblemen der amerikanischen Gesellschaft gegenuber der hispanischen Einwanderergruppe sehen. Die Vorbehalte richten sich vor allem gegen einen neuen spanischsprachigen Abgeordnetenblock, der sich noch weniger als die Vertreter Hawaiis an den politischen Hauptinteressen des amerikanischen Festlandes orientieren würde. Ein neuer Bundesstaat brächte überdies eine Neuberechnung der Sitzverteilung mit sich. So hätte Puerto Rico Anspruch auf zwei Senats- und sechs Abgeordnetensitze, die an die Stelle bisheriger Repräsentanten aus anderen Landesteilen träten und zudem den "hispanischen Stimmenblock" im Kongreß verstärken würden. Im KongreB haben daher die Faktoren der Sprachenregelung und der sogenannten "super-ma jority" die Bedeutung von Prüfsteinen für die Puerto Rico-Entscheidung angenommen 13 .

12 Vgl. José-Luis Mendez: "Une nouvelle intitative de Washington dans les Caraibes - Vers un statut de "République Associée" pour Porto-Rico", in: Le Monde Diplomatique, Januar 1987.

13 Die Annexion der spanischsprachigen Insel wünde über kur oder lang die Frage der offiziellen Landessprache der USA aufwerfen. Und ein Beitritt zur Union ist in allen historischen Präzedenzfällen von einer sogenannten Supermehrheit im Beitrittsplebiszit abhängig gemacht worden; eine einf ache 51 \%ige Anschlußmehmeit wurde bisher nie als ausreichend anerkannt Das Votum Puento Ricos im November 1993 hat daher den amerikanischen Kongreß von außerordentlichen Entscheidungsproblemen befreit. Vgl. hierzu die interessante Analyse von Barreto M., Amilcar A.: "The Debate over Puerto Rican Statehood: Language and the super-majority", in: Revista Homines (San Juan), Bd. 16 (1991/92) 2, S. 135-41. 
Die Schwankmgen im Engagement der amerikanischen Exekutiven in Sachen Puerto Rico sind natülich bis zu einem gewissen Grade ein Reflex der Stimmungslage in den verschiedenen Parteifraktionen des Kongresses und den wirtschaftspolitischen Pressure Groups der USA. In groben Zügen läßt sich die Interessenverteilung so umreißen, daß die nordamerikanischen Landwirtschaftsinteressen grundsätzlich eine Integration der Insel - wegen der hinderlichen Import-Schutzklauseln - eher befüwortet haben. Die Industrieuntemehmerschaft - soweit sie Geschäftsbereiche vertritt, fur deren Investitionen die Insel Steuerpräferenzen bietet - war stets gegen die Integration, da diese eine Verlagerung der Anlagen in andere Billiglohnländer ausgelöst hätte. Beide Interessenbereiche sind stärker in der Republikanischen Partei vertreten als bei den Demokraten, doch hat die Republikanische Fraktion im Kongreß insgesamt eher ein Bild der Zurdickhaltung als eines der Befürwortung puertoricanischer Integration vermittelt.

In der Demokratischen Partei spielen gewerkschaftliche Einflüsse eine bedeutende Rolle; im Gegensatz zur puertoricanischen Gewerkschaftsbewegung herrscht hier traditionell die Befürwortung des Beitritts zur Union vor. Hinzu kommt, daß die Demokratische Partei traditionell ein Sammelbecken der sogenarmten ethnic vote, d.h. auch der Stimmen hispano-amerikanischer Einwanderer geworden ist. Generell entsteht daher der Eindruck, daß die Parteibasis der Demokraten tendenziell zur Integration neigt, während die Republikaner mehrheitlich die Beibehaltung des bisherigen Statuts fur Puerto Rico befürworteten.

Die Stimmungslage in den US-Parteien ist somit seit Jahren nicht in voller Ubereinstimmung mit der jeweils befreundeten Partei in Puerto Rico verlaufen, so daß sich in der Statusfrage eine Art O'ber-Kreuz-Allianz ergeben hat. Gouverneur Rosello (PNP) hat denn auch vor dem Volksentscheid stärker auf die Unterstiutzung der Demokraten gesetzt, als auf die der ihm programmatisch näherstehenden Republikanischen Partei14.

Eine weitere Dimension hat die amerikanische Politik in der Statusfrage allerdings in den letzten Jahrzehnten dadurch erhalten, daß die Präsidenten nahezu regelmäßig vom Meinungstrend der eigenen Partei abgewichen sind. Die republikanischen Präsidenten Reagan und Bush waren Befürworter der Eingliederung Puerto Ricos, während Präsident Carter eine sehr distanzierte Haltung einnahm, wie sie sehr ähnlich auch im zurückhaltenden Engagement Präsident Clintons in der Kampagne 1993 zum Ausdruck kam. So läßt sich eine auf zwei Ebenen uber Kreuz verlaufende Unterstützungsallianz erkennen, nach deren Muster die amerikanische Exekutive jeweils die puertoricanische Partnerpartei unterstiutzte, sich darin aber von der Meinungslage in der eigenen US-Partei abhoben. Ubergeordnete innenpolitische Beweggründe geben hier offensichtlich den Ausschlag, und das wesentlichste Element ist dabei , welchen Einfluß die Puerto Rico-Regelung auf die sogenannte "hispanic vote", d.h. das Abstimmungsverhalten der wachsenden spanischsprachigen Wäh- 
lerschaft, in den US-Wahlen hätte. Maßgebend war für Präsident Bush und das Republican National Committee die Oberlegung, daß ein puertoricanischer Bundesstaat mit entsprechender hispanischer Vertretung in Repräsentantenhaus und Senat die Wirkung auslösen könnte, den spanischsprachigen Wæhlerstamm aus seiner traditionellen Loyalitätsbindung zur Demokratischen Partei zu lösen 15 und somit einer für die Republikaner abträglichen demographischen Entwicklung in der Wählerschaft der Vereinigten Staaten entgegenzuwirken.

\section{Strittige Vorschläge zur Erweiterung de Commonwealth-Statuts im Vorfeld des Volksentscheides}

Nachdem die Frage des Beitritts zur Union einige Jahre lang auf der Insel sozusagen in einem Vakuum weitergefuhr worden war, ethielt die Debatte ab 1986 neuen Auftrieb aus den USA. Die Regienmg Reagan setzte sich zu Beginn ihrer zweiten Amtszeit eine generelle Neueinschätzung und "Bereinigung" der vertraglichen Bindungen mit ihren Treuhandgebieten zum Ziel. Dies betraf in erster Linie die pazifischen Inselterritorien, deren völkerrechtlicher Status ständig vor den Vereinten Nationen angemahnt worden war. Als Ergebnis wurde nach 17-jährigen Verhandlungen im Januar 1986 mit den Marshall-Inseln und den Federated States of Micronesia ein neuer "Compact of Free Association" abgeschlossen16. Aber auch der Fall Puerto Rico wurde in diesem Zusammenhang vom Innenausschuß des Kongresses (in dessen Zustăndigkeit Treuhand- und Assoziienungsgebiete stehen) wieder aufgegriffen und zwischen April und Juli 1986 zum Gegenstand von drei Reihen offentlicher Anhörungen gemacht.

In Vorbereitung der Statusverhandlungen des Kongresses hatte 1986 der Rechnungshofpräsident (Comptroller General/General Accounting Office) einen Zustandsbericht über Puerto Rico vorgelegt 17 , der für die Insel den erweiterten Status einer "assoziierten Republik" zur Diskussion stellte - eine Lösung etwa auf der Mitte zwischen dem bisherigen Status und der Bundesstaatlichkeit. Bereits die Autorenschaft dieses Modells deutet an, da $B$ in Washington

15 Siehe: Time Magazine, 26.03.1990, S. 32.

$16 \mathrm{Zu}$ den völkerrechtlichen und inhalt lichen Aspekten dieser Statusverhandlungen vgl. von Krosigk, Friedrich: "Die Auflösung kolonialer Herrschaft im Süd-Pazifik", in: ders./G. Rath/W. Leithold: Südsee-Inselwelt im Umbruch", Erlangen 1988, S. 66-82. Mit Palau haben sich ähnliche Statusverhandlungen weiter hingezogen und konnten erst im November 1993 nach einem ähnlichen Status-Plebiszit mit einem "Compact of Free Association" abgeschlossen wenden.

17 Comptroller General: Experiences of Past Territories Can Assist Puerto Rico Status Deliberations (Repor No. GGD 80-26), Washington, 07.03.1980. 
zunehmend Erwägungen über die fiskalische Belastung eine Rolle spielten, die das gegenwärtige Commonwealch-Statut Puerto Ricos furr die USA mit sich bringt18.

Auf der Insel lösten die Washingtoner Statusdebatte und die vorgeschlagene Republiklösung hochgehende Wogen politischer Erregung aus. Keine der drei entscheidenden politischen Gruppen entschloB sich jedoch zu einer Befurwortung dieses Modells, und zwar vor allem, weil es einen schrittweisen Abbau der finanziellen Subventionen vorsah. Lediglich der autonomistische Flügel "Pro-ELA" innerhalb des PPD und einige berufsständische Verbände traten für die vom US-Rechnungshof zur Diskussion gestellte Autonomieerweiterung ein. Im erhitzten politischen Klima von San Juan konnte sich nun aus der Opposiionspartei PNP, unter Führung der Ex-Gouverneure Romero Barceló und Ferré, eine Plattform für den Beitritt zur Union bilden, die ab 1988 mehr und mehr Massenrückhalt gewann. Da auch die Unabhängigkeitspartei PIP darauf vertraute, ihren Stimmenanteil auf mindestens $12 \%$ ausweiten zu können, rechneten sich alle drei politischen Gruppen Erfolgschancen bei der Abstimmung aus, so daB erstmals eine konsensuale Ausgangsbasis für die Abhaltung eines, international anerkannten Volksentscheids gegeben war. Gemeinsam wurde im Januar 1989 beim KongreB in Washington die Zustimmung zu diesem endgültigen Statusplebiszit beantragt. Der US-Senat handelte nun rasch, und aus seinen Anhörungen im Frühsommer 1989 ging nicht nur die efforderliche Zustimmung zum Volksentscheid hervor, sondem auch eine Festlegung der Konditionen für jede der drei Statusoptionen. Besonders das Modell einer assoziierten Republik, als Fortentwicklung des ELA-Statuts, wurde dabei genauestens eingegrenzt, so daB der Eindruck einer indirekten Befürwortung der Integrationsoption entstehen konnte. Verschreckt trat nun die regierende PPD unter Gouverneur Hernández19 eine Flucht nach vom an und entwickelte zum Vorschlag der "assoziierten Republik" ein Gegenmodell der autonomeren Ausgestaltung des bestehenden Statuts und setzte zugleich für Ende 1991 ein Sonderreferendum uber eine Charta der Bürgerrechte (Charter of Rights) an, die die wesentlichen Elemente der vorgeschlagenen Statusregelung enthielt (bei einer dem Republikmodell ähnlichen Autonomiegestaltung sollten im wesentlichen die finanziellen Leistungen der USA für alle Zukunft beibehalten werden). Auf diese Weise versuchte die puertoricanische Regienung, gegenüber dem Modell des US-Rechnungshofs unter dem Schlagwort eines "ELA culminado" (etwa:

18 Die Subventionen und Transferleistungen der USA an Puerto Rico beliefen sich 1992 auf rund $\$ 8$ Mrd.; davon getrennt erfolgen die Zahlungen der Sozialfürsorge des Bundes (Empfänger gegenwärig 1,35 Mio Personen = 37,5\% der Inselbevölkerung). Allein das Lebensmittel-Coupon-Programm umfaßt monatliche Leistungen i. H. v. \$ 199 pro Person und Monat, d. h. weitere rund \$ 3,3 Mrd.; hier ist bis 1996 eine Erhöhung des Monatssatzes auf \$ 331 vorgesehen. Im Gegenzug argumentierte die frühere PPD-Regierung mit Berechnungen, die bei der Eingliederung in die USA bis zum Jahr 2000 eine etwa doppelt so hohe jährliche Belastung des Bundesstaats mit \$25 Mrd. erwarten lassen (Time Magazine, 26.03.1990, S. 32).

19 Gouvemeur Hemández Cólón lehnteden Senatsvorschlag als "terribly dangerous and unacceptably unbalanced" ab (Time Magazine, 26.03.1990). 
"die Vollendung des Freistaats") ein eigenes erweitertes Commonwealth-Modell durchzusetzen 20. Mit der erwarteten Zustimmung zu dieser Agenda im Referendum 1991 - so das Kalkül - wäre nicht nur die Verhandlungsposition gegenüber Washington gestärkt sondem eine bestimmende Vorentscheidung fur das Statusplebiszit getroffen worden.

Mit dieser Strategie ist die damalige Regienung gescheitert, denn das Referendum vom Dezember 1991 ergab eine unerwartet hohe Ablehnung des Regienngsvorschlags (53\% zu $43 \%$ ) und damit auch der Verhandlungsvollmacht. Der Ausgang dieser Volksbefragung besiegelte praktisch die Erfolgsaussichten des PPD fur ein erneutes Mandat bei der Parlaments- und Gouverneurswahl im November 1992 und veranlaBte Gouverneur Hemandez, das vorgesehene Plebiszit zu vertagen. Nach dem Sieg des oppositionellen PNP 1992 hat erst Gouvemeur Pedro Roselló den Volksentscheid neu angesetzt, im Vertrauen auf einen Sieg der bundesstaatlichen Option.

Abgesehen von den zur Wahl stehenden Extremlösungen (staatliche Unabhängigkeit oder Beitritt zur Union) sind fur Puerto Rico somit wăhrend der jungeren politischen Entwicklung drei Modelle fitr eine daverhafte Autonomieregelung unter formalem Beibehalten des Commonwealth-Statuts vorgebracht worden und jeweils verworfen worden:

- "Compact of Permanent Union" (Nixon/Ford, 1976)

- "Associated Republic" (Rechnungshof, 1986, mit Spezifizierung im Entwurf des Senats von Juni 1989)

- "Estado Libre asociado culminado" (= "enhanced Commonwealch status", Hernandez Cólón, 1991).

Keiner der drei Vorschläge zur Umgestaltung oder Ausgestaltung des bisherigen Status hat sich zwischen den beiden Verhandlungsseiten als konsensfähig erwiesen. Dabei waren die ersten beiden Entwürfe aus dem Blickwinkel der US-Interessen gestaltet und hatten zum Angelpunkt eine schrittweise Übertragung der finanziellen Eigenverantwortlichkeit an Puerto Rico (bei entsprechender Reduzierung der Belastung für die USA), während das dritte Modell die puertoricanische Interessenlage zum Inhalt hatte und auf weitestmögliche völkerrechtliche Handlungsfähigkeit Puerto Ricos unter Beibehaltung aller Leistungen und Garantien von seiten der USA gerichtet war.

Durch das Ergebnis des Volksentscheids ergibt sich eine Situation, in der sich erstmals die Vertreter der beiden großen Optionen in annăhernd gleich großen Blöcken gegenüberstehen, ohne jedoch eine für den US-KongreB uberzeugende oder gar bindende Mehrheit erzielt zu haben. Ein weiterer Aspekt, der ermeute Statusverhandlungen erschweren dürfte, liegt darin, daß zwar 1993 formal die staatsrechtliche Option der puertoricanischen Oppo-

20 Vgl. hierzu im Detail: Daniel, Justin: "Porto Rico - Système politique et comportements électoraux", in: Problèmes d'Amérique Latine No. 99 (1991), S. 31-39. 
sition (also: Beibehaltung des ELA-Statuts) obsiegt hat, der dahinter verborgenen Zielsetzung der Ausweitung dieses Statuts im Verlauf weiterer Verhandlungsrunden mit den USA jedoch im Referendum vom Dezember 1991 das Vertrauen der Mehrheit versagt worden war. Die jetzige und die künfuge puertoricanische Regierung verfügen somit zunächst über kein Mandat zu weiteren Fordenungen an die USA. Diese sind ihrerseits weiter an die bestehende staatsrechtliche Regelung und die damit verbundenen finanziellen Lasten gebunden, von denen sie einseitig kaum Abstriche machen kömnen21. PNP und die Befürworter des Beitritts zur Union verweisen zwar auf ihren stetig gewachsenen Rückhalt in der puertoricanischen Bevölkenung, aber eine Wiedervorlage der Frage zu emeuter Entscheidung ist fir sie vorerst ausgeschlossen; ihr wirde in absehbarer Zeit auch eine emeute Zustimmung des Kongresses versagt bleiben. Puerto Rico und die Vereinigten Staaten werden sich darauf einrichten müssen, daß die Konditionen der gegenseitigen Bindung auf längere Frist festgeschrieben sind und der Spielraum für Verändenungen durch weitere Verhandlungen eingeengt bleibt.

Eine massive Verschiebung der Meinungslage in künfigen Wahlen zu den puertoricanischen Vertretungskörperschaften (die dam uberzeugende Schubkraft in Washington für einen Neuansatz auslösen würde) ist eigentlich nur unter der Voraussetzung einer grundlegenden Terrainverschiebung in den Handels- und Investitionsbedingungen des mittelamerikanischen-karibischen Raumes insgesamt vorstellbar. So könnte sich etwa aus einer dynamischen Entwicklung der nordamerikanischen Freihandelszone NAFTA und ihrer Ausstrahlung auf die karibischen Staaten unter CBI-Präferenz ein weiterer dramatischer Schwund der Wettbewerbsvorteile der puertoricanischen Sonderstellung ergeben, der dann den Anstoß zu einem grundsätzlichen Überdenken des bestehenden Vertragswerks mit sich brächte.

21 Die wichtigsten Punkte, bei denen die USA Einschränkungen gegenüber Puerto Rico durchzusetzen sucht, betreffen die Verwaltungshoheit für den Umweltschutz der Korallenriff-Küste und das Steuerpräferenzprogramm nach Section 936 der US-Steuergesetzgebung. Seit 1980 wurde dreimal versucht, die Steuerpräferenz abzuschaffen oder zu modifizieren; mit der absehbaren Folge eines Abbaus von rd. 300.000 der industriellen Arbeitsplätzen auf der Insel. 1987 wurde in der Kontroverse ein Stillhalteabkommen erreicht. Vgl. "Puerto Rico told 936 is now safe", in: Latin American Weekly Report RC-87-08, 01.10.1987. Dennoch hat der US-Congress im Frühjahr beschlossen, daß die Steuerpräferenz unter Section 936 ab Ende 1994 schrittweise abgebaut werden soll (Time Magazine, 08.1 1.93, S. 48.) 
The Dynamics of Civilization of the State and of the Generation of Baroque Politics in Black Africa

By Luc Sindjoun

The current political changes in francophone Black Africa have deep repercussions in the constitutional order, respite the permanence of certain inhibitants of democratic devclopment. In fact, francophone Black Africa seems to be the showground of the emergence of a janus-headed State, piecing together in a chaotic way the long authoritarian continuance and the liberal instant. In the framework of this article, it will be attempted to relativize the paradigm of the historicity of authoritarianism in light of the most recent political tendencies in Africa, and to confine the paradigm of liberal optimism, being aware of the inherent difficulty of an absolute fracture on the socio-political field. This accounts for the pertinence of the epistemology of baroque politics.

\section{The Puerto Rican Status Issue}

\section{By Ulrich Fanger}

The question of eventual independence for Puerto Rico has been a politically sensitive issue for over 50 years. In response to the quest for a definitive settlement, the Puerto Rican electorate has twice been called to a referendum in order to determine the intemational status of the island; previously, six vote calls since 1898, as well as two specific plebiscites (in 1970 and 1991) had had the sovereignty question as their central issue.

The present article traces the constitutional development of the island on its way towards commonwealth status (established in 1950) and examines the political developments, both on the domestic and the intemational scene, that were instrumental in bringing the stat us debate to the forefront again. Particular attention is devoted to the various conseculive proposals put forward over the years to redefine the statutory bond between Puerto Rico and the U.S. mainland, and to the reasons why they were rejected. 